\title{
An Investigation into 2D and 3D Shapes Perception
}

\author{
Vanja ČOK, Daria VLAH, Roman ŽAVBI
}

\begin{abstract}
Numerous research results support the finding that a product's visual appearance is important. In particular, end products or services that have a direct productuser interaction have to be developed in accordance with the taste of the user and the market. The user-centered product should be designed and created according to both the technical requirements and the customer needs, but it should also differentiate itself from the competition. The shape of a product, whether we observe it in 2D or 3D, should communicate various intangible meanings. Our aim was to investigate whether the meaning of bipolar adjectives varies when observing samples of 2D and 3D shapes. The study was conducted using 2D shape contour samples and interactive 3D extruded models that could be rotated in virtual space. In order to determine the relationship between the shape and the user's perception of it, Kansei engineering methodology was used. We collected data with Semantic differential survey using five level Likert scale. The results revealed minor deviations in the users' perceptions of the 2D and 3D sample shapes.
\end{abstract}

Keywords: 2D shape; 3D shape; Product Design; Shape perception

\section{INTRODUCTION}

Many studies on customer products have shown that a user's perception of a product's design influences the decision of the product purchase. Unfortunately, a user's reaction to a novel product is often very difficult to predict. The more novel a product is, the higher the level of its incongruity, and the more difficult it is for user to understand and appreciate a product's value [1]. This is particularly so when the user has to recognise a product's usefulness based only on a visual impression, without having any previous experience of it. Product design is a combination of functional, symbolic and aesthetic dimensions that defines how an individual will react to the product [2, 3]. Basically, all design disciplines combine knowledge of aesthetics with the knowledge of different embodying technologies [4]. Designers are therefore using diverse approaches to generate a product's intangible meanings. According to Nagamachi [5] sophisticated consumers desire products which match their own feelings of design, function and price. In order to achieve the desired design of a product we first need to be familiar with the influencing visual elements (line, shape, pattern, texture, colour, tone, form, space) of the observed object to better understand how the user's perception can be manipulated. Visual perception is usually described with the theories of formal aesthetic rules that define the basic principles, such as ratio, harmony, a desire for consistency and other principles in order to create pleasant visual stimuli [6]. Skillful creators use and manipulate aesthetic rules when designing a product in order to induce pleasant impressions with the users. In an early stage of the product development process when designers draw 2D sketches to present their initial ideas, they are simultaneously developing a product-design "language". Product design language or design vocabulary is a specific style that guides the design of a complement of products or architectural settings [7]. It is risky to make decisions about product appearance based on initial design sketches and drafts because all the following design phases will be affected by it. To mitigate these risks researchers use innovative approaches to draw user's attention to the product's design [8-12]. However, customer's psychological feeling evoked by a product appearance is complex to measure. To approach this issue several consumer's satisfaction evaluation methods have been developed such as Quality Function Deployment (QFD), Conjoint Analysis and Voice of Customer, but they do not consider the association of customers' needs with product design characteristics [13]. A recognized tool for exploring user's psychological feeling (impression) towards the product design (i.e. parameters) is Kansei engineering [5]. Kansei represents an individual's subjective impression from certain artifact, environment or situation that includes the senses of sight, hearing, feeling, smell, taste and the sense of balance, as well as recognition [14]. As kansei is a person's subjective and unstructured feeling towards something and therefore cannot be measured directly, we use indirect measurement methods that consider alternative forms of expression [15]. Since kansei covers a diversity of expressions that range from psychological to psychophysiological, many different tools and techniques have been developed to measure them [13].

There are two streams in the research of shape perception. One investigates how consumers make preference judgments while taking into account both the product's form and its function [16]. The other one specializes in the user's perception with respect to a simplified product's visual elements such as abstract shapes or shape contours [17]. The principle of reducing a product's visual elements, or the so-called abstraction, is often applied in the preparation of shape samples. In most cases the methodology of Kansei engineering uses pictures of final products for measuring user's feedbacks [11].To gain more comprehensive understanding of how the user perceives objects based on the type of representation, we have explored interaction with 2D and 3D shapes.

In engineering design shapes and contours are used in the early development phases to visualize ideas, concepts, etc. 3D models are used in the later phases (embodiment and detail design) when the product structure is already established. 2D drawing on paper is considered to be quicker and much easier than 3D modeling [18]. In this case, shape contours provide a source of information about the 3D structure of an object i.e preliminary product layout [19]. In engineering design 3D CAD models are based on 2D sketches. 
The interest of our study is user's perception of main visual characteristics of 2D and 3D shape samples, which are usually designed in the early design phase. In our study user is the person who directly interacts with the presented shape. The findings of the study could help designers and engineers understand how they should present product ideas to future users (potential customers).

\section{BACKGROUND}

Abstraction is a broad term or concept used in many research areas. The essence of creating abstraction is to reduce the complexity of the problem [20]. The process of abstraction means the generalization of the existing concept, while at the same time preserving the relevant information or purpose. It is used to assist creative processes and problem solving because it prevents the fixation on conventional and obvious ideas, but it also focuses on the core of the task and new solutions [21]. In the development of a product, abstraction in the early design stage is used to achieve inventiveness and innovation [22]. Reduction of details in the context of product aesthetics or so called "product geometrization" was presented by Lewalski [23]. According to Bouchard et al. [9] simplified shape contours or silhouettes among other visual materials (texture, colors, patterns, layouts) are a source of inspiration for product designers. They have formalized design process in order to create kansei information system and defined the relations that designers use to establish the various levels of abstraction of information in a design process. They refer to this definition as kansei ontology, where these levels of information go from very abstract values, such as purity, to concrete attributes, such as the colour type, for example: white. A definition of low-level information is that it encompasses the characteristics of shape, colour and texture, while middle-level information includes concepts and artefact names, and high-level information is translated by semantic descriptors, sociological values and affective and emotional dimensions [9]. If we refer to Gestalt psychology, the main aspect is that the mind understands external stimuli as a whole and not as the sum of the individual parts $[24,25]$. The geometrization of visual samples is important for simplifying its perception. Even for purely organic forms, we can detect a geometrical arrangement if we do not focus on the details. There are additional advantages of using geometric shapes, e.g. in the manufacturing process for industrial products, geometric shapes are preferred because they simplify the production process.

\subsection{Formalisation of 2D and 3D Shapes (Shape Grammar)}

In order to formalise shape and its features we have to understand how to define what shape is and how it is observed in similar studies. The basic elements of a shape are the points, lines and plots [26]. The line is the base from which all other design symbols originate and can represent geometric shapes such as square, circle, triangle, ellipse etc. Forms from solid bodies are one of the fundamental elements of the third dimension [27]. Usually, the design must follow predefined rules and laws [27]. On the basis of Gestalt principles and the theory of form as a whole, in
1971 a Shape grammar was developed [26]. Shape grammar serves to study two or three dimensional shapes which are defined by 2D and 3D language [27, 28, 12, 29] Studies of Shape grammar are more oriented towards investigating a formal inventory of compositions, i.e., the relationships between shapes in the case where the user's preferences regarding shapes are not included. Currently, in most cases the evaluations of products attractiveness involve the pictures of finished products instead of the real objects, concept representations or interfaces [30]. This limits the possibilities to influence the design during the conception process. To summarize, the existing studies mostly focused on investigating user's shape preferences with respect to $2 \mathrm{D}$ sample shapes, where parameters such as the curvature, the contour curve, the silhouette, the colour and texture of the shape, or the wavelet transformation and Fourier transformation were typically observed [31].There is a lack of research that compares the influences of shape representation, such as $2 \mathrm{D}$ and $3 \mathrm{D}$ representation, on user's perception of an object. Tavanti and Lind [32] have investigated the effects which 2D and 3D displays have on user's spatial memory. Their research revealed positive effects of the three-dimensional representations of information on the users. Despite such efforts, a more thorough research on the effects of $3 \mathrm{D}$ representational models is still needed. Presumably, the main reason for the scarcity of data about 3D shape perception could be that it is complicated to cover and to define a shape's characteristics in 3D space.

\subsection{Shape Semantics}

When designing a user-oriented product the geometrical shape becomes one of the most important factors, together with other visual factors such as colour, material and texture [33]. Besides its functionality, visual appearance of a product is influenced by socio-cultural, socio-economic, historical and technological factors. According to the Gestalt theorists, preferences regarding the shape of a product should be affected by a person's already innate sense of proportion or ratio [34]. Certain lines, ratios, shapes and colours are inherently attractive [35]. A complex product design, without the coherence of visual elements, usually induces complex cognitive processing [36]. The majority of people prefer the shapes that moderately stimulate their attention, for example, Wundt curves. Moderate irregularities and uncertainties in product shapes are closer to humans [25]. Preferences about shapes depend on the memories and experiences that an individual has with a particular subject [37]. Researchers use different criteria for determining human preferences for the shape of a product [38-41, 23, 10, 4248]. Most frequently researchers use three categories to describe a cognitive response to product appearance: aesthetic impression, semantic interpretation and symbolic association [38, 39]. Numerous studies involving various cases of everyday products indicate that people generally prefer rounded to linear lines, surfaces and shapes [17, 49, 44]. Sharp edges metaphorically threaten because such objects are often dangerous [50, 51]. It has also been confirmed that angular objects cause greater stimulation of the amygdala than the curved objects $[17,52]$. Shape characteristics are also dependent on fashion trends. 
Blijlevens et al. [46] noted that in the 1980s products with straight lines were considered to be modern, while at present, organic forms are perceived as more modern [46]. Many studies have shown deviations of human perception toward diverse shapes and their meanings. Consumers associate their feelings toward product design by using words or adjectives like elegant, sportive etc. In Kansei engineering a semantic differential technique is used for investigating perceived meaning of the observed object [11]. In order to clarify relationship between an adjective (semantic) and form element scientists have also developed different shape categorizations and named product form types. Users are more likely to understand the product if they can categorize it or put it within an existing group. In their measurement of emotional responses Chang and $\mathrm{Wu}$ [53] found that fourteen different properties are categorized within five types of product forms, which they named as aesthetic, biological, cultural, novel and conceptual forms. According to their research results, aesthetic and biological forms of products have a greater potential, as they attract the attention of a younger group of customers and evoke pleasure. Leder and Carbon [44] studied the characteristics of the interior of a car with three design variables represented by complexity, curvature and innovation. Hsiao and Chen [49] conducted semantic differential study on 88 chairs in which they measured trendiness, complexity and emotion by using sets of bipolar adjectives (traditional-modern, simple-complex and rational-emotional) and evaluated novelty and aesthetic preference with additional set of bipolar adjectives (typical-unique and beautiful-ugly).

The relationship between the product criteria and the customer's satisfaction is linear, e.g., if the customer perceives the product as being of high quality, then the customer's satisfaction is higher, and vice versa [54] In the previous studies people's impression of the gender of shapes was measured using the semantic association of adjectives "feminine/masculine". They found that the gender of a 3D geometrical shape is influenced by the variations of its base, axis and sweep [33]. In many studies, researchers have investigated user's perception toward shapes but focused on semantic meanings and not so much on the shape sample representations. In this paper, we present a new approach to investigate differences in perception of 2D and 3D shapes. Since previous studies are focused on semantic meanings related to product's 2D visualizations (pictures, contours), in our research we tend to investigate two types of shape sample representations (2D and $3 \mathrm{D}$ ) in relation to five product characteristics described in chapter 4.1 .

\section{RESEARCH AIM}

Our aim was to discover whether there is any difference in the associations and the meanings users attach to shapes when observing them in 2D and 3D space. Based on the literature preview we assume that 3D shapes will be more descriptive and will have more representational power than 2D shapes [32]. In addition, shapes with curved corners are according to study of Bar and Neta [17] more preferred in comparison to shapes with angular corners. Our interest was to discover if the change of shape representation from $2 \mathrm{D}$ to $3 \mathrm{D}$ space will result in different perception of their meaning. In order to explore our research aim we have posed two research questions:

Q1: Do 3D shapes have higher scores attached to adjectives in comparison to 2D shape contours?

Q2: Do 3D shapes with curved corners differentiate by attached meaning in comparison to the same samples presented in $2 \mathrm{D}$ space?

\section{METHOD}

Based on Kansei engineering methodology we selected the product samples and defined the affective dimensions (the product's intangible characteristics). Ten samples of shapes contours were used in this survey and represented in black and white colour in order to avoid the influence of colour on the participant's perception (Fig. 3). The shape contour characteristics (2D) were taken from pellet-burner designs (3D). We collected pictures of pellet burners from different promotional magazines, literature and from the design team. Then the product's pictures and later the shape contours were converted from $2 \mathrm{D}$ into a $3 \mathrm{D}$ extruded model. The sample shapes were simplified using the principles of geometrization based on Lewalski's theory of elements reduction [23]. After the procedure of geometrization, the elements concerning the shape were classified into two categories: straight and curved cornered shapes. We then prepared questionnaires based on Semantic differential technique and 5-level Likert scale (described under point 4.1). In the questionnaires shape samples were randomly arranged. In order to find the differences or similarities among the 2D and 3D shape samples we compared the mean values using Independent t-test statistics.

\subsection{Metrics of Affective Dimensions}

Shape semantics will be represented by a defined product characteristics. We defined four product characteristics that were observed during the experiment (Fig. 1). This research resulted from an industrial case related to redesign of pellet burners, therefore the outline was on functionality attributes. Each of the product characteristics contained bipolar adjectives that serve to measure the effective meaning of a single shape. The adjectives were collected from different sources and literature. We have adopted aesthetics preference (adj. beautiful-ugly) and complexity preference (adj. simplecomplex) from Hsiao and Chen [49]. We have renamed complexity preference (simple-complex) to functional attribute. Future attribute (futuristic-unfuturistic) has replaced a novelty preference proposed by Blijlevens et al. [46]. We have added a social attribute (femininemasculine) and functional attribute with additional bipolar adjectives (reliable-unreliable, uncomfortable-comfortable and simple-complex). All the selected adjectives were general, used in everyday life and understandable to all the participants. Afterwards, they were arranged with respect to the product-dimension categories (Fig. 1).

The subject used a 5-point Likert scale to rate the shape's aesthetic attribute $(1=$ very ugly, $5=$ very beautiful), the shape's functional attribute $(1=$ very uncomfortable, 5 = very comfortable), the shape's social attribute ( $1=$ very feminine, $2=$ very masculine $)$, the 
shape's functional attributes $(1=$ very unreliable, $5=$ very reliable), $(1=$ very complex, $5=$ very simple $)$ and the shape's futuristic attributes (very unfuturistic - very futuristic). We will use mean values as an indicator of the intensity of feedbacks.

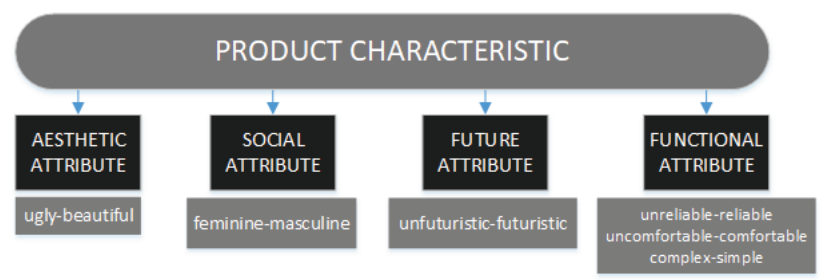

Figure 1 Four main product characteristics

\subsubsection{Samples}

The aim of the geometrization is to transform a real product or sketch into an abstraction (Fig. 3). To obtain an initial idea of how the users perceive separate product elements it is necessary to have a variety of different shapes [55]. We have selected 8 pellet-burners examples from promotional magazines and literature available on internet. Additionally, we have added 2 pellet burners designs which were created by the design team in our laboratory (shape $f$ and shape $j$ ). Four samples had straight and six samples had rounded corners. We decided not to interfere with the shapes acquired from the sources, so that the case would resemble to what can be found in the real environment. Criteria for selection of pellet-burners was a diversification among the samples. We have asked two experts from the product domain to help us with the selection of samples.

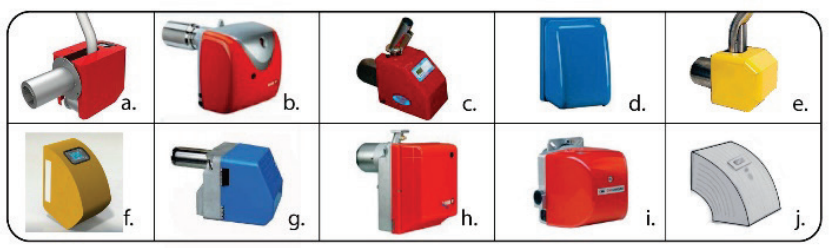

Figure 2 Examples of pellet burners that formed the basis for $2 \mathrm{D}$ shape contours

Shape contours were extracted from a side view of pellet-burners samples (Figs. 2, 3). Based on the shape contour, we have prepared 2D sketches for CAD models.

\section{from product to abstract shape}

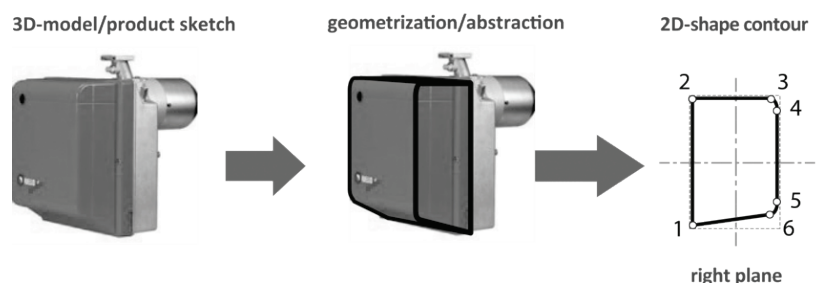

Figure 3 Preparation of samples (from product to abstract shape)

For a clearer data interpretation we divided these samples into two categories based on the angularity of their shapes (Fig. 4). The criteria for the separation were straight or curved corners. In this experiment we intentionally did not associate the shape with any shape symbolism.

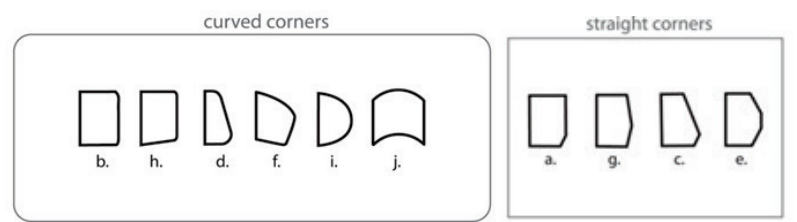

Figure 4 Sets of angular and rounded sample shapes

3D shape samples were extruded based on the 2D sketches using CAD software Solidworks 2014 version (Fig. 5).

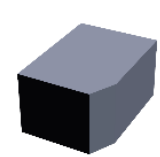

a

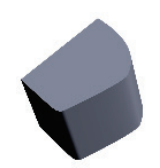

f

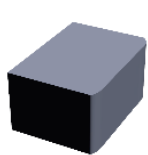

b

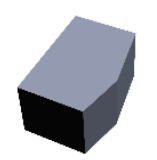

g

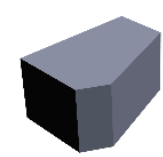

C

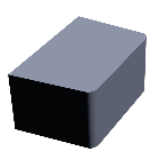

$\mathrm{h}$

Figure 5 3D shape samples

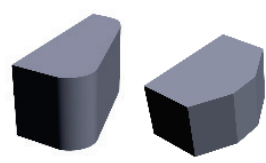

d

e

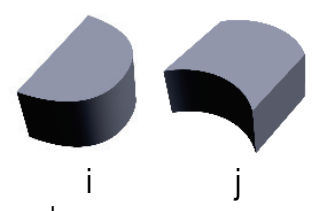

\subsubsection{Participants}

The experiment involved engineering students. A total of 45 male students aged 20-30 were invited to take part in the interactive survey. They were involved in the same study programme. Their educational background was in mechanical engineering and the participants were familiar with the use of CAD software.

\subsubsection{Procedure}

The questionnaire was divided into three parts. The first part dealt with demographic questions related to gender, age, place of residence and education. The second part of the questionnaire examined the emotional response of the students to 2D shapes using the semantic differential technique according to Osgood [56]. A 5-level Likert scale was used to rate the samples of $2 \mathrm{D}$ shapes associated to bipolar adjectives. In the third part every student had to give feedback to an interactive presentation of a 3D shape (3D pdf) on a stationary computer (Fig. 5). The survey was conducted in computer-based classrooms during the day. The participants received paper questionnaires and clear instructions. Participants had to fill in the demographic questions in the first page. Next, on the paper below each picture of a 2D shape contour was a semantic differential scale where the participant had to rate his feeling about bipolar adjective with connection to the 2D shape at 5-level scale. Then, they had a semantic differential questionnaire with the same bipolar adjectives in Excel 2014 with an active link to interactive $3 \mathrm{D}$ model in pdf. In order to avoid boredom effect among participants of the study we have changed the order of bipolar adjectives. For the statistical analysis we transposed the values and changed the order of the bipolar pairs together with the values, e.g. (1) beautiful - (5) ugly to (1) ugly - (5) beautiful.

The size of LCD display was 21.5 " across the diagonal. The students were allowed to move, rotate and zoom in or zoom out the 3D model and observe the model from different angles. 


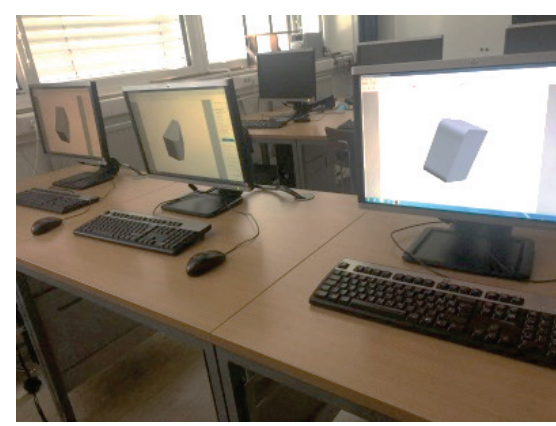

Figure 6 Shape of questionnaire with an active link to 3D Shape mode

\section{RESULTS}

First, we performed the descriptive and then inferential statistical analysis. With the Independent $t$-test, we compared means of scores of two independent group samples and investigated the difference in the participants responses. The independent $t$-test is done with the assumption that the variances of the two groups being measured are equal.

A statistical analysis was performed using SPSS version 22 , while the statistical significance was set at $p<$ 0.05 .

First we have explored if 3D shapes evoke higher scores attached to adjectives in comparison to $2 \mathrm{D}$ shape contours. Comparing 2D and 3D shape samples we have received the result that demonstrates no statistically significant difference in scores given to $2 \mathrm{D}$ shapes $(M=$ $3.05, \mathrm{SD} .5120)$ and to $3 \mathrm{D}$ shapes $(M=3.06, S D=.0786)$ with $t(118)=-.072, p=.942$.

Second, when comparing curved cornered samples, 2D and 3D shapes we again learned that there is no statistically significant difference with $t(70)=.061, p=.952 .2 \mathrm{D}(M=$ $3,16 ; S D=.518), 3 \mathrm{D}(M=3,15 ; S D=.609)$.

Generally, for both research questions no statistically significant differences among 2D and 3D shapes were found. We have decided to observe separate comparisons using Independent-t test for each 2D and 3D shape sample in relation to bipolar adjective. We have discovered a few statistically significant differences among them. In the following synthesis only the results with statistically significant differences will be described. The results are sorted in terms of shape samples.

Rounded 2D and 3D shapes:

Shape D:

Statistically significant differences were found when observing the adjective beautiful-ugly, where the 2D shape $(M=3,29 ; S D=1.100)$ and the 3D shape $(M=3,84 ; S D=$ $0.852)$ were perceived as beautiful with $t(88)=-2.679, p$ $=.009$ (Fig. 7).

\section{Shape H:}

Evoked almost neutral feedback but with consideration of standard error it was scored as ugly in the 2D presentation $(M=2.93 ; S D=.863)$ while the $3 \mathrm{D}$ shape $(M$ $=3.40 ; S D=.939)$ was perceived as beautiful with the statistical significance $t(88)=-2.454, p=.016$.

For shape $\mathrm{H}$ and the bipolar adjectives comfortableuncomfortable the participants responses were almost neutral for 2D shape $(M=3.09 ; S D=.874)$ but again with consideration of standard error they were scored as comfortable the same as 3D shape $(M=3.51 ; S D=1.079)$ with $t(88)=-2.039, p=.044$.

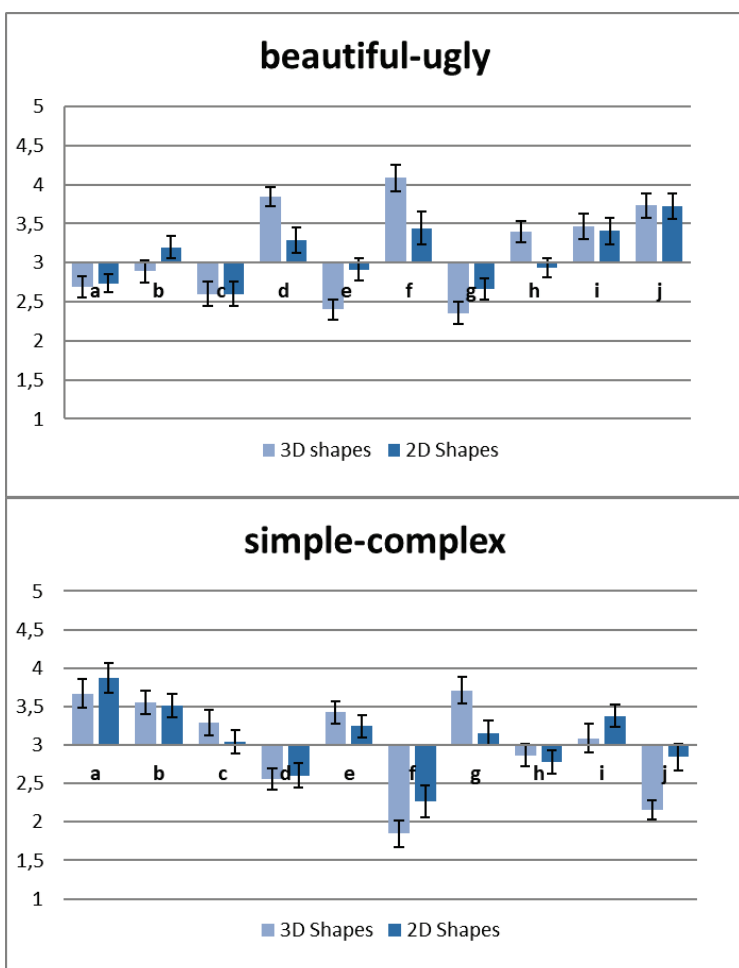

Figure 7 Comparison of 2D and 3D shapes according to Kansei words beautifulugly (above) and simple complex (below)

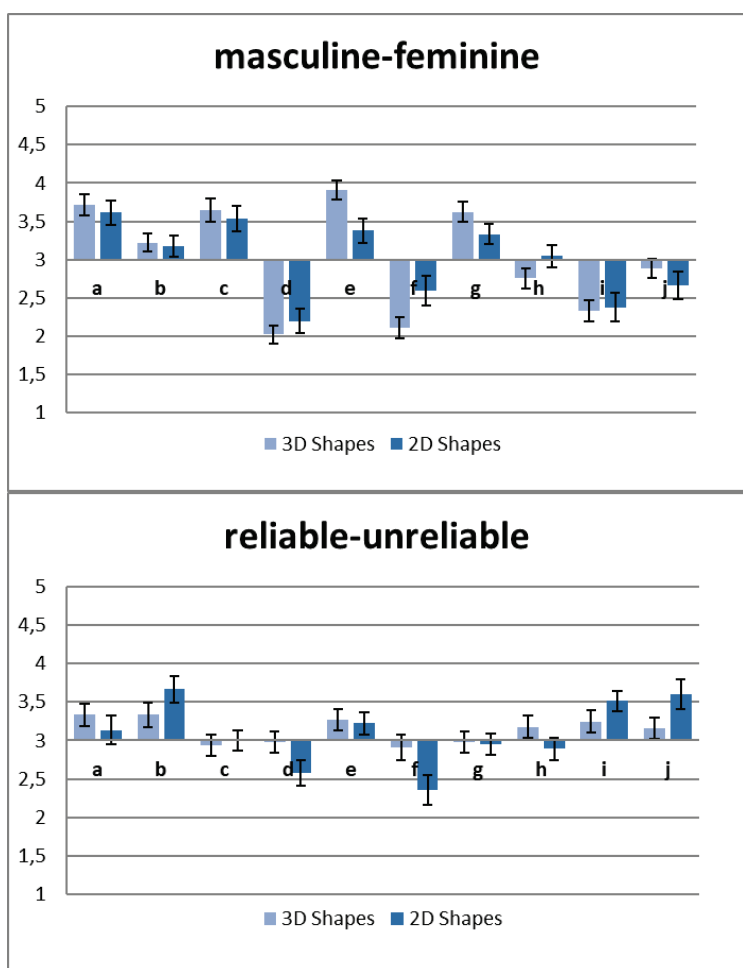

Figure 8 Comparison of the 2D and 3D shapes according to the Kansei words masculine-feminine (above) and reliable-unreliable (below)

Shape F:

It was perceived as beautiful for both the 2D shape ( $M$ $=3.44 ; S D=1.358)$ and the $3 \mathrm{D}$ shape $(M=4.09 ; S D=$ $1.145), t(88)=-2.434, p=.017$. 
It was assumed to be feminine in both $2 \mathrm{D}(M=2.60$; $S D=1.304)$ and 3D $(M=2.11 ; S D=.935)$ presentations with $t(88)=2.044, p=.044$ (Fig. 8).

In both the $2 \mathrm{D}(M=2.36 ; S D=1.300)$ and $3 \mathrm{D}(M=$ 2.91; $S D=1.145)$ presentations shape $\mathrm{F}$ was perceived as unreliable with $t(88)=-2.152, p=.034$.

Shape I:

It was perceived as comfortable both in $2 \mathrm{D}(M=3.89$; $S D=1.153)$ and $3 \mathrm{D}(M=3.36 ; S D=1.264)$ presentations, with $t(88)=2.091, p=.039$.

Shape J:

It was considered complex in both $2 \mathrm{D}(M=2.84 ; S D$ $=1.205)$ and $3 \mathrm{D}(M=2.16 ; S D=.852)$ with $t(88)=3.131$, $p=.002$. Again, equal variances were not assumed (Fig. $7)$.

At the bipolar adjective comfortable-uncomfortable scores for 3D sample $(M=3.09 ; S D=1.145)$ were almost neutral but considered as comfortable as well as at the $2 \mathrm{D}$ sample $(M=3.80 ; S D=1.198)$ with $t(88)=2.878, p=.005$ (Fig. 9).

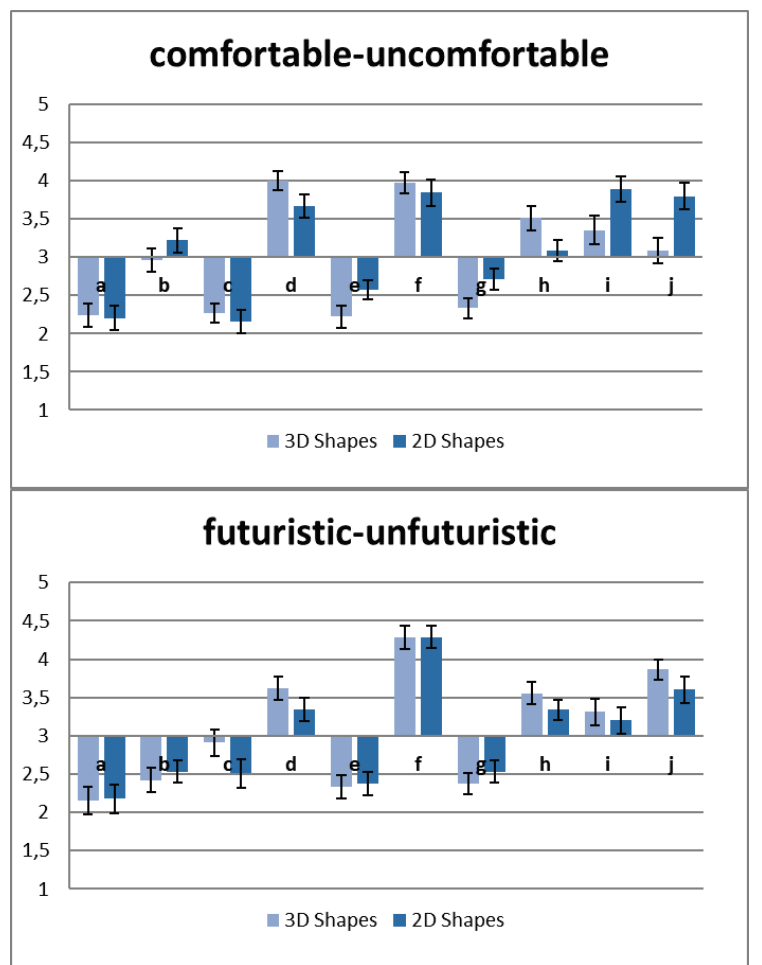

Figure 9 Comparison of the 2D and 3D shapes according to the Kansei words comfortable-uncomfortable (above) and futuristic-unfuturistic (below)

Angular shapes 2D and 3D:

Shape G:

It was considered simple both for $2 \mathrm{D}(M=3,16 ; S D=$ $1.043)$ and $3 \mathrm{D}(M=3,71 ; S D=1.141)$ with $t(88)=-2.411$, $p=0.018$.

\section{Shape E:}

It was perceived to be ugly in both the 2D shape $(M=$ $2,91 ; S D=.973)$ and 3D shape samples $(M=2,40 ; S D=$ $.889), t(88)=2.601, p=.011$. Equal variances were not assumed because Levene's test for equality of variances was statistically significant.
It was perceived as masculine in both $2 \mathrm{D}(M=3.38$; $S D=1.072)$ and 3D $(M=3.91 ; S D=.848)$, with $t(88)=$ $-2.617, p=.010$. Equal variances were not assumed.

\section{DISCUSSION}

Our interest was to explore whether the participants will attach different meanings while observing 2D and 3D shape samples with curved corners. We also investigated whether a group of participants would give higher scores attached to adjectives to $3 \mathrm{D}$ shapes in comparison to $2 \mathrm{D}$ shapes. Survey included 4 main product characteristic (6 bipolar adjectives) and 10 shape samples among which 4 samples had straight corners and 6 had curved corners.

According to our research question Q1 we have found that there is no statistically significant difference in scores attached to adjectives when comparing $2 \mathrm{D}$ and $3 \mathrm{D}$ shape samples. Therefore, 3D shapes did not evoke as much higher scores in comparison to 2D shapes as we expected.

When we tested our research question Q2 we have found that curved cornered 2D shapes and 3D shapes do not evoke different associations among our participants since there was no statistically significant difference between perceived bipolar adjectives (product characteristics) in relation to shape samples.

After testing the hypotheses we have observed separate 2D and 3D shape samples in relation to bipolar adjectives. The most common difference found was a range in the intensity of emotional responses (difference between lowest and highest mean value at the same adjective). In most cases 3D shapes have slightly higher means in comparison to $2 \mathrm{D}$ shapes but not to all the observed adjectives. It is interesting that in the comparison between the $2 \mathrm{D}$ and $3 \mathrm{D}$ shapes, the $3 \mathrm{D}$ shapes $(\mathrm{H}, \mathrm{F})$ are having higher scores at adjective "beautiful".

In summary, the main deviations in the meanings attached to shapes were found among the rounded shapes (F, H, D, J, I). Shape H was percieved as ugly in 2D but as beautiful in 3D presentation. From a group of rounded shapes no statistically significant differences were found for shape B, comparing it in 2D and 3D space. Statistically significant differences among angular shapes were found for shape $G$ and shape E. Again, rounded shapes were associated with more positive adjectives in comparison to angular shapes and rounded 3D shapes have had a slightly higher mean values in comparisons to $2 \mathrm{D}$ shapes.

The results have shown that for four shapes (A, G, C, B) there were no statistically significant differences. It is interesting that for bipolar adjective "unfuturisticfuturistic" also no statistically significant deviations in meaning comprehension among participants were found. Participants have had the same opinion on shapes that are futuristic. Most deviations in scores were found at shape F. Other researchers that have investigated people's impression about the gender of shapes in terms of semantic association "feminine/masculine" found that the gender of a $3 \mathrm{D}$ geometric shape is influenced by the variations of its base, axis and sweep [33]. They found that the gender of a $3 \mathrm{D}$ shape is highly dependent on the curved contours of its base. We have found that except for the shape B, rounded shapes were percieved as feminine. Certainly, specific shape elements such as shape corners have an impact on the meaning attached to the shape. 
As mentioned previously, the results of our study demonstrate that between the $2 \mathrm{D}$ and $3 \mathrm{D}$ shape samples less differences were found than we expected. Accoring to our results participants s perception of $3 \mathrm{D}$ shapes only slightly differed from 2D shapes. Thus we cannot conclude that 3D shapes have had greater representional power, as it was the case in the reasearch conducted by Tavanti and Lind [32]. Participants were united in the meanings attached to straight cornered shapes (A, G, C) while curved cornered shapes caused minor deviations. According to Bar \& Neta [17] curved designs are more preferred than straight designs and curvature elicits positive feedbacks. This could be the explanation that most of the participants focused more on the estimation of rounded shapes. Since designers use both 2D sketches and 3D models to present their ideas, perhaps they could simplify the represenation by using side views of the model in order to recieve quick feedback from the users. Our results support the findings from Koenderink and Van Doorn [19] that shape contours could provide a source of information about the $3 \mathrm{D}$ structure of an object.

\section{CONCLUSION}

It is possible that the differences in the users' perception would be greater if a chamfer or fillet feature was added to the 3D samples. The limitation of our research was that the group of participants were only males. Perhaps having a more gender balanced sample of participants would also influence the final result. It is important to understand the semantic meaning and the possible limitations of these findings. We have intentionally isolated observed shape samples from the context of use and any practical function in order to receive clearer insight into differences based on abstract level of $2 \mathrm{D}$ and 3D shapes. Findings of our study imply that user's perception would not change whether the design of basic product shape is presented as $2 \mathrm{D}$ sketch or $3 \mathrm{D}$ model. Our intention was to investigate product visual characteristics based on abstract and simplified shape samples. Results of this study can serve to designers who are developing product design language using simplified sketches. In future studies it would be interesting to consider the context of the product's use so that the participants would have the possibility to relate the shape with something familiar. In addition, for future studies we suggest the use of interactive representations of the shape samples in a virtual space.

\section{Acknowledgements}

The research was funded by Slovenian Research Agency (ARRS) No. P2-0256.

\section{REFERENCES}

[1] Petkova, A. \& Rindova, V. (2006). When is a New Thing a Good Thing? Technological Change, Product Form Design and Perceptions of Value for Product Innovations, Design Research Society, International Conference in Lisbon (IADE). https://doi.org/10.1287/orsc.1060.0233

[2] Pratt, M. G. \& Rafaeli, A. (2006). Artifacts and Organizations: Understanding our "objective" reality. In A. Rafaeli and M. Pratt (Eds.), Artifacts and Organizations:
Beyond Mere Symbolism. Mahwah, NJ: Lawrence Erlbaum Associates.

[3] Rafaeli, A. \& Vilnai-Yavetz, I. (2004), Instrumentality, aesthetics and symbolism of physical artifacts as triggers of emotion. Theoretical Issues in Ergonomics Science, 5, 91112. https://doi.org/10.1080/1463922031000086735

[4] Hung, W. K. \& Chen, L. L. (2012). Effect of Novelty and its Dimensions on Aesthetic preference in Product Design. International Journal of Design, 6(2), 81-90.

[5] Nagamachi, M. (1995). Kansei Engineering: A new ergonomic consumer-oriented technology for product development. International Journal of Industrial Ergonomics, 15, 3-11. https://doi.org/10.1016/0169-8141(94)00052-5

[6] Arnheim, R. (1974). Art and Visual Perception: A Psychology of the Creative Eye, University of California Press.

[7] Brunner, R., Emery, S., \& Hall, R. (2009). Do You Matter?: How Great Design will Make People Love Your Company. Pearson Education, 157-72.

[8] Tsuchiya, T., Nagamachi, M., \& Yukihiro Matsubara, Y. (2005). Kansei Engineering for Design of Basic 3D Rectangular. Proceedings of the 2005 International Conference on Active Media Technology, (AMT 2005). https://doi.org/10.1109/AMT.2005.1505394

[9] Bouchard, C., Gentner, A., \& Esquivel, D. (2014), About the nature of Kansei information, from abstract to concrete. KEER 2014, Linkoping, June 2014. International conference on Kansei engineering and Emotion research. https://hal.archives-ouvertes.fr/hal-01097238

[10] Schütte, S. (2005). Engineering Emotional Values in Product Design, Kansei Engineering in Development, Linköping.

http://www.diva-portal.org/smash/record.jsf?pid=diva2\%3A20839

[11] Bongard-Blanchy, K. (2013). Bringing the user experience to early product conception: From idea generation to idea evaluation. PhD thesis. Other. Ecole nationale supérieure d'arts et métiers - ENSAM, 2013. English. <NNT : 2013ENAM0068>. <pastel-00936893>

[12] Cagan, J. (2001). Engineering Shape Grammars: Where Have We Been and Where are We Going? In: Antonsson, E. K. \& Cagan, J. (eds). Formal Engineering Design Synthesis. Cambridge University Press, Cambridge, UK https://doi.org/10.1017/CB09780511529627.006

[13] Lokman, A. M. (2009). Emotional user experience in Web Design: The Kansei engineering approach, Doctoral Thesis, December 2009.

[14] Nagamachi, M.(2002), Kansei engineering as a powerful consumer-oriented technology for product development. Applied Ergonomics, 33289-294, Elsevier. https://doi.org/10.1016/S0003-6870(02)00019-4

[15] Lévy, P. (2013). Beyond kansei engineering: The emancipation of kansei design. International Journal of Design, 7(2), 83-94.

https://research.tue.nl/en/publications/beyond-kansei-engineering-the-emancipation-of-kansei-design(ecd4175d-98f7-4e51-a0d5$51 \mathrm{c} 5 \mathrm{ccded} 4 \mathrm{f3}) \cdot \mathrm{htm}$

[16] Sylcott, B., Tabibnia, G., \& Cagan, J. ( 2011). Understanding of emotions and reasoning during consumer tradeoff between function and aesthetics in product design, Proceedings of the ASME International Design Engineering Technical Conferences \& Computers and Information in Engineering Conference IDETC/CIE 2011, August 28-31, 2011, Washington, DC, USA https://pdfs.semanticscholar.org/8426/be3a210a6307c9be1a2987 2e182d1e2e0e32.pdf

[17] Bar, M. \& Neta, M. (2006). Humans prefer curved visual objects. Psychological Science, 17(8), 645-648. https://doi.org/10.1111/j.1467-9280.2006.01759.x 
[18] Gingold, Y., Igarashi, T., \& Andzorin, D. (2009). Structured annotations for 2D-to-3D modeling. ACM Transactionson Graphics (TOG), 28(5), 1-9. https://doi.org/10.1145/1618452.1618494

[19] Koenderink, J. \& van Doorn, A. J. (1982). The Shape of Smooth Objects and the Way Contours End. Perception, 11, 129-137. https://doi.org/10.1068\%2Fp110129

[20] Whittlesea, B. W. A. (1983). The representation of concepts: an evaluation of the abstractive and episodic perspectives. $P h D$ thesis, McMaster University, Hamilton, Canada. http://hdl.handle.net/11375/5985

[21] Pahl, G., Beitz, W., Feldhusen, J., \& Grote, K. H. (2007). Engineering Design: A Systematic Approach, (K. Wallace \& L. Blessing, Trans. Third edition.) Darmstadt, Germany: Springer-Verlag London. https://doi.org/10.1007/978-1-84628-319-2

[22] Duhovnik, J. (1990). Contribution for natural classification of products related to $\mathrm{CAD} / \mathrm{CAM}, \mathrm{CIRP}$ International Seminar on CA-Design, Ljubljana.

[23] Lewalski, Z. M. (1988). Product Esthetics: An interpretation for Designers, Carson City, NV: Design \& Development Engineering Press.

[24] Ellis, W. D. (1950). A Sourcebook of Gestalt Psychology. New York: The Humanities Press.

[25] Jones, P. L. (1991). Taste Today, New York: Pergamon Press. https://philpapers.org/rec/JONTTT-5

[26] Stiny, G. \& Gips, J. (1972). Shape grammars and the generative specification of painting and sculpture. In Information Processing, 71, 1460-1465. North-Holland Publishing Company. http://www.cs.bc.edu/ gips/ShapeGrammarsIFIPS71.pdf

[27] Stiny, G. (2006). Shape: Talking about Seeing and Doing. MIT Press, Cambridge, MA. https://doi.org/10.7551/mitpress/6201.001.0001

[28] Chen, W., Wiecek, M., \& Zhang, J. (1999). Quality UtilityA Compromise Programming Approach to Robust Design. J. Mech. Des., 121(2), 179-187. https://doi.org/10.1115/1.2829440

[29] Chau, P. Y. K., Cole, M., Massey, A. P., Montoya-Weiss, M., \& O'Keefe, R. M. (2002). Cultural differences in the online behavior of consumers. Communications of the ACM, 45(10), 138-143. https://doi.org/10.1145/570907.570911

[30] Bongard-Blanchy, K., Bouchard, C., \& Aoussat, A. (2013). Limits of Kansei - Kansei unlimited. International Journal of Affective Engineering, 12(2), 145-153. https://doi.org/10.5057/ijae.12.145

[31] Mi Choi, S. \& Yong-Guk, K. (2005). Similarity Estimation of 3D Shapes Using Modal Strain Energy, R. Khosla et al. (Eds.): KES 2005, LNAI 3683, 206-212. Springer Verlag, Berlin Heidelberg. https://doi.org/10.1007/11553939_30

[32] Tavanti, M. \& Lind, M. (2001). 2D vs 3D, implications on spatial memory. IEEE Symposium on Information Visualization, INFOVIS 2001. https://doi.org/10.1109/INFVIS.2001.963291

[33] Jayasinghe, H., Rajapakse, R. P. C. J., \& Miyata, K. (2008). Analysis of 3D shapes by gender kansei. In Proc. of the Second International Conference on Kansei Engineering and Affective Systems (KEAS'08), Nagaoka, Japan, Nov. 2224, pp. 129-134.

[34] Doczi, G. (1981). The Power of Limits, Boulder, Co: Shambala Publications.

[35] Arnheim, R. (1992). But is it science? G. C. Cupchik and J. Laszlo' (eds) Emerging visions of the aesthetic process: psychology, semiology, and philosophy, Cambridge University Press, Cambridge, UK, 27-36.

[36] Durgee, J. F. (1988). Product Drama. Journal of Advertising, 17 (February/March), 42-49. https://psycnet.apa.org/record/1989-35000-001

[37] Leymarie, F. \& Kimia, B. (2001). The Shock Scaffold for Representing 3D Shape. Proc. of $4^{\text {th }}$ International Workshop on Visual Form (IWVF4). Published in "Visual Form 2001", Lecture Notes in Computer Science (LNCS 2059), SpringerVerlag, 216-229. https://doi.org/10.1007/3-540-45129-3_19

[38] Norman, D. A. (2002). Emotion \& design: attractive things work better. Interactions, IX(4), 36-42. https://doi.org/10.1145/543434.543435

[39] Crilly, N., Moultrie J., \& Clarkson, P. J. (2004). Seeing things: consumer response to the visual domain in product design. Design Studies, 25(6). https://doi.org/10.1016/j.destud.2004.03.001

[40] Jordan, P. W. (2000). Designing pleasurable products: an introduction to the new human factors, Taylor \& Francis Books Ltd, UK. https://doi.org/10.4324/9780203305683

[41] Tiger, L. (1992). The Pursuit of Pleasure. Boston: Little, Brown, and Co.

[42] Hsu, S. H., Chuang, M. C., \& Chang, C. C. (2000). A semantic differential study of designers and users product form perception. International Journal of Industrial Ergonomics, 25, 375-391. https://doi.org/10.1016/S0169-8141(99)00026-8

[43] Creusen, M. E. H. \& Schoormans, J. P. L. (2005). The Different Roles of Product Appearance in Consumer Choice. Journal of Product Innovation Management, 22(1), 63-81. https://doi.org/10.1111/j.0737-6782.2005.00103.x

[44] Leder, H. \& Carbon, D. (2005). Dimensions in Appreciation of Car Interior Design. Applied Cognitive Psychology, 19, 603-618. https://doi.org/10.1002/acp.1088

[45] Blijlevens, J., Creusen, M. E. H., \& Schoormans, J. P. L. (2009). How consumers perceive product appearance: The identification of three product appearance attributes. International Journal of Design, 3(3), 27-35.

[46] Blijlevens, J., Mugge, R., Ye, P., \& Schoormans, J. P. L. (2013). The influence of product exposure on trendiness and aesthetic appraisal. International Journal of Design, 7(1), 55-67.

[47] Batra, R. (1990). Measuring the hedonic and Ulitarian Sources of Consumers Attitudes. Marketing letters, 2(2), 159-170. https://doi.org/10.1007/BF00436035

[48] Hassenzahl, M. (2004). The Interplay of Beauty, Goodness, and Usability in Interactive Products. Human-Computer Interaction, 19(4), 319-349. https://doi.org/10.1207/s15327051hci1904_2

[49] Hsiao, K. A. \& Chen, L. L. (2006). Fundamental dimensions of affective responses to product shapes. International Journal of Industrial Ergonomics, 36(6), 553-564. https://doi.org/10.1016/j.ergon.2005.11.009

[50] Berlyne, D. (1970). Novelty, complexity and hedonic value. Perception and Psychophysics, 8, 279-286. https://doi.org/10.3758/BF03212593

[51] Berlyne, D. (1976). The new experimental aesthetics and the problem of classifying work of art. Scientific Aesthetics, 1, 85-106.

[52] Silvia, P. J. \& Barona, C. M. (2009). Do people prefer curved objects? Angularity, expertise, and aesthetic preference. Empirical Studies of the Arts, 27, 25-42. https://doi.org/10.2190/EM.27.1.b

[53] Chang, W. C. \& Wu, T. Y. (2007). Exploring types and characteristics of product forms. International Journal of Design, 1(1), 3-14.

[54] Chen, C. C. \& Chuang, M. C. (2008). Itegrating the Kano model into robust design approach to enhance customer satisfaction with product design. Int. J. Production Economics, 114(2), 667-681. https://doi.org/10.1016/j.jpe.2008.02.015

[55] Čok, V. \& Duhovnik, J. (2016). Exploration of users' crosscultural differences by using the Kansei engineering approach: chapter 6. V: FUKUDA, Shuichi (ed.). Emotional engineering. Vol. 4. Cham [etc.]: Springer. 1-26. https://doi.org/10.1007/978-3-319-29433-9_6 
[56] Osgood, C. E., Suci, G. J., \& Tannenbaum, P. H. (1957). The Measurement of Meaning. University of Illinois Press: USA.

\section{Contact information:}

Vanja ČOK, PhD, Teaching Assistant

University of Ljubljana,

Faculty of Mechanical Engineering,

Aškerčeva 6, 1000 Ljubljana, Slovenia, Europe

E-mail: vanja.cok@lecad.fs.uni-lj.si

Daria VLAH, PhD student, Researcher

University of Ljubljana,

Faculty of Mechanical Engineering,

Aškerčeva 6, 1000 Ljubljana, Slovenia, Europe

E-mail: daria.vlah@lecad.fs.uni-lj.si

Roman ŽAVBI, PhD,Full professor

University of Ljubljana,

Faculty of Mechanical Engineering,

Aškerčeva 6, 1000 Ljubljana, Slovenia, Europe

E-mail: roman.zavbi@lecad.fs.uni-lj.si 